\title{
Strong convergence of a new general iterative method for variational inequality problems in Hilbert spaces
}

Yan-Lai Song ${ }^{1,2}$, Hui-Ying Hu', Ya-Qin Wang ${ }^{3}$, Lu-Chuan Zeng ${ }^{1,4^{*}}$ and Chang-Song Hu²

\author{
* Correspondence: zenglc@hotmail. \\ com \\ 'Department of Mathematics, \\ Shanghai Normal University, \\ Shanghai 200234,China \\ Full list of author information is \\ available at the end of the article
}

\begin{abstract}
In this article, we introduce a new iterative scheme with Meir-Keeler contractions for strict pseudo-contractions in Hilbert spaces. We also discuss the strong convergence theorems of the new iterative scheme for variational inequality problems in Hilbert spaces. The methods in this article are interesting and are different from those given in many other articles. Our results improve and extend the corresponding results announced by many others.

MR(2000) Subject Classification: 47H09; 47H10.

Keywords: Hilbert space, k-strict pseudo-contractions, fixed point, Meir-Keeler contractions
\end{abstract}

\section{Introduction}

Let $H$ be a real Hilbert space with inner product $\langle\cdot, \cdot\rangle$ and norm $\|\cdot\|$. Let $C$ be a nonempty closed convex subset of $H$ and let $F: C \rightarrow C$ be a nonlinear operator. The variational inequality problem is such that

$$
V I(F, C): \quad\left\langle F x^{*}, v-x *\right\rangle \geq 0, \quad \forall v \in C .
$$

Variational inequalities were introduced and studied by Stampacchia [1] in 1964. It is now well known that variational inequalities cover as diverse disciplines as partial differential equations, optimal control, optimization, mathematical programming, mechanics and finance, see [1-25].

It is known that if $F$ is a strongly monotone and Lipschitzian mapping on $C$, then the $\operatorname{VI}(F, C)$ has a unique solution. It is also known that the $\operatorname{VI}(F, C)$ is equivalent to the fixed point equation

$$
x^{*}=P_{C}\left[x^{*}-\mu F\left(x^{*}\right)\right],
$$

where $P_{C}$ is the projection of $H$ onto the closed convex set $C$ and $\mu>0$ is an arbitrarily fixed constant. So, fixed point methods can be implemented to find a solution of the $V I(F, C)$ provided $F$ satisfies some conditions and $\mu>0$ is chosen appropriately. A great deal of effort has gone into finding an approximate solution of the $V I(F, C)$ see $[3,5,15-19]$.

\section{Springer}

(C) 2012 Song et al; licensee Springer. This is an Open Access article distributed under the terms of the Creative Commons Attribution License (http://creativecommons.org/licenses/by/2.0), which permits unrestricted use, distribution, and reproduction in any medium, provided the original work is properly cited. 
In 2001, Yamada [2] introduced the following hybrid iterative method for solving the variational inequality

$$
x_{n+1}=T x_{n}-\mu \lambda_{n} F\left(T x_{n}\right), n \geq 0 .
$$

On the other hand, Yao et al. [6] modified Mann's iterative scheme by using the socalled viscosity approximation method which was introduced by Moudafi [7]. More precisely, Yao et al. [6] introduced and studied the following iterative algorithm:

$$
\left\{\begin{array}{l}
x_{0}=x \in C \\
y_{n}=\beta_{n} x_{n}+1\left(1-\beta_{n}\right) T x_{n} \\
x_{n+1}=\alpha_{n} f\left(x_{n}\right)+\left(1-\alpha_{n}\right) y_{n}, n \geq 0 .
\end{array}\right.
$$

where $T$ is a nonexpansive mapping of $K$ into itself and $f$ is a contraction on $K$. They obtained a strong convergence theorem under some mild restrictions on the parameters.

Zhou [8], Qin et al. [9] modified normal Mann's iterative process (1.3) for non-self- $k$ strictly pseudo-contractions to have strong convergence in Hilbert spaces. Qin et al. [9] introduced the following iterative algorithm scheme:

$$
\left\{\begin{array}{l}
x_{1}=x \in K \\
y_{n}=P_{K}\left[\beta_{n} x_{n}+\left(1-\beta_{n}\right) T x_{n}\right] \\
x_{n+1}=\alpha_{n} f\left(x_{n}\right)+\left(1-\alpha_{n} A\right) y_{n}, n \geq 1 .
\end{array}\right.
$$

where $T$ is non-self- $k$-strictly pseudo-contraction, $f$ is a contraction and $A$ is a strong positive linear bounded operator. They prove, under certain appropriate assumptions on the sequences $\left\{\alpha_{n}\right\}$ and $\left\{\beta_{n}\right\}$, that $\left\{x_{n}\right\}$ defined by (1.4) converges strongly to a fixed point of the $k$-strictly pseudo-contraction, which solves some variational inequality.

The following famous theorem is referred to as the Banach contraction principle.

Theorem 1. (Banach [10]) Let $(X, d)$ be a complete metric space and let $f$ be a contraction on $X$, i.e., there exists $r \in(0,1)$ such that $d(f(x), f(y)) \leq r d(x, y)$ for all $x, y \in X$. Then $f$ has a unique fixed point.

Theorem 2. (Meir and Keeler [11]) Let $(X, d)$ be a complete metric space and let $\varphi$ be a Meir-Keeler contraction (MKC) on $X$, i.e., for every $\varepsilon>0$, there exists $\delta>0$ such that $d(x, y)<\varepsilon+\delta$ implies $d(\varphi(x), \varphi(y))<\varepsilon$ for all $x, y \in X$. Then $\varphi$ has a unique fixed point.

Remark 1. Theorem 2 is one of generalizations of Theorem 1, because contractions are MKCs.

Question 1. Can Theorem 1 of Yao [6], Theorem 3.2 of Zhou [8], Theorem 2.1 of Qin [9], and so on be extended from one or finite $k_{i}$-strictly pseudo-contraction to infinite $k_{i}$-strictly pseudo-contraction?

Question 2. We know that the MKC is more general than the contraction. What happens if the contraction is replaced by the MKC?

Question 3. We know that the $\eta$-strongly monotone and $L$-Lipschitzian operator is more general than the strong positive linear bounded operator. What happens if the strong positive linear bounded operator is replaced by the $\eta$-strongly monotone and $L$-Lipschitzian operator?

Question 4. Can the restrictions imposed on the parameters $\left\{\alpha_{n}\right\},\left\{\beta_{n}\right\}$ and $\left\{\lambda_{n}\right\}$ in [9] be relaxed? 
The purpose of this article is to give the affirmative answers to these questions mentioned above. Motivated by the above works, in this article we suggest and analyze a hybrid iterative algorithm as follows:

$$
\left\{\begin{array}{l}
x_{1}=x \in C \\
y_{n}=P_{C}\left[\beta_{n} x_{n}+\left(1-\beta_{n}\right) \sum_{i=1}^{\infty} \mu_{i}^{(n)} T_{i} x_{n}\right] \\
x_{n+1}=\alpha_{n} \phi\left(x_{n}\right)+\gamma_{n} x_{n}+\left(\left(1-\gamma_{n}\right) I-\alpha_{n} F\right) y_{n}, n \geq 1 .
\end{array}\right.
$$

where $T_{i}$ is a non-self- $k_{i}$-strictly pseudo-contraction, $\varphi$ is an MKC contraction and $F$ : $C \rightarrow C$ is a $L$-Lipschitzian and $\eta$-strongly monotone mapping in Hilbert space. Under certain appropriate assumptions on the sequences $\left\{\alpha_{n}\right\},\left\{\beta_{n}\right\},\left\{\gamma_{n}\right\}$, and $\left\{\mu_{i}^{n}\right\}$, that $\left\{x_{n}\right\}$ defined by (1.5) converges strongly to a common fixed point of an infinite family of $k_{i^{-}}$ strictly pseudo-contractions, which solves some variational inequality.

\section{Preliminaries}

In this section, we first recall some notations. Let $C$ be a nonempty closed convex subset of a real Hilbert space $H$. Let $F: C \rightarrow C$ be an operator. $F$ is called $L$-Lipschitzian if there exists a positive constant $L$ such that

$$
\|F x-F y\| \leq L\|x-y\|,
$$

for all $x, y \in C, F$ is said to be $\eta$-strongly monotone if there exists a positive constant $\eta$ such that

$$
\langle F x-F y, x-y\rangle \geq \eta\|x-y\|^{2},
$$

for all $x, y \in C$. Without loss of generality, we can assume that $\eta \in(0,1]$ and $L \in$ $[1, \infty)$. Under these conditions, it is well known that the variational inequality problem $V I(F, C)$ has a unique solution $x^{*} \in C$.

A self-mapping $f: C \rightarrow C$ is a contraction on $C$ if there exists a constant $\alpha \in(0,1)$ such that $\|f(x)-f(y)\| \leq \alpha\|x-y\| ; \forall x, y \in C$. We use $\Pi_{C}$ to denote the collection of all contractions on $C$. That is, $\Pi_{C}=\{f f: C \rightarrow C$ a contraction $\}$. We use $F(T)$ to denote the fixed point set of the mapping $T$ and $P_{C}$ to denote the metric projection of $H$ onto its closed convex subset $C$.

A mapping $T$ is said to be non-expansive, if

$$
\|T x-T y\| \leq\|x-y\| \quad \text { for all } x, y \in C .
$$

$T$ is said to be a $k$-strict pseudo-contraction in the terminology of Browder and Petryshyn [12], if there exists a constant $k \in[0,1)$ such that

$$
\|T x-T y\|^{2} \leq\|x-y\|^{2}+k\|(I-T) x-(I-T) y\|^{2}, x, y \in C .
$$

It is clear that it is equivalent to

$$
\langle T x-T y, x-y\rangle \leq\|x-y\|^{2}-\frac{1-k}{2}\|(I-T) x-(I-T) y\|^{2}, x, y \in C,
$$

or is equivalent to

$$
\langle(I-T) x-(I-T) y, x-\gamma\rangle \geq \frac{1-k}{2}\|(I-T) x-(I-T) y\|^{2}, x, y \in C
$$


An operator $A$ be a strongly positive bounded linear operator on $H$, that is, there exists a constant $\bar{\gamma}>0$ such that

$$
\langle A x, x\rangle \geq \bar{\gamma}\|x\|^{2}, \forall x \in H .
$$

Remark 2. From the definition of $A$, we note that a strongly positive bounded linear operator $A$ is a $\|A\|$-Lipschitzian and $\bar{\gamma}$-strongly monotone operator.

In order to prove our main results, we need the following lemmas.

Lemma 2.1 (Zhou [8]). Let $H$ be a Hilbert space and $C$ be a closed convex subset of $H$. If $T$ is a $k$-strictly pseudo-contractive mapping on $C$, then the fixed point set $F(T)$ is closed convex, so that the projection $P_{F(T)}$ is well defined.

Lemma 2.2 (Zhou [8]). Let $H$ be a Hilbert space and $C$ be a closed convex subset of $H$. Let $T: C \rightarrow H$ be a $k$-strictly pseudo-contractive mapping with $F(T) \neq \emptyset$. Then $F$ $\left(P_{C} T\right)=F(T)$.

Lemma 2.3 (Browder and Petryshyn [12]). Let $H$ be a Hilbert space, $C$ be a closed convex subset of $H$, and $T: C \rightarrow H$ be a $k$-strictly pseudo-contractive mapping. Define a mapping $J: C \rightarrow H$ by $J x=\alpha x+(1-\alpha) T x$ for all $x \in C$. Then, as $\alpha \in[k, 1), J$ is a non-expansive mapping such that $F(J)=F(T)$.

Lemma 2.4. (see [13]). Let $\left\{x_{n}\right\},\left\{z_{n}\right\}$ be bounded sequences in a Banach space $E$ and $\left\{\beta_{n}\right\}$ be a sequence in [0,1] which satisfies the following condition: $0<\lim \inf _{n \rightarrow \infty} \beta_{n} \leq$ $\lim \sup _{n \rightarrow \infty} \beta_{n}<1$. Suppose that $x_{n+1}=\left(1-\beta_{n}\right) x_{n}+\beta_{n} z_{n}$ for all $n \geq 0$ and $\limsup _{n \rightarrow \infty}$ $\left(\left\|z_{n+1}-z_{n}\right\|-\left\|x_{n+1}-x_{n}\right\|\right) \leq 0$. then $\lim _{n \rightarrow \infty}\left\|z_{n}-x_{n}\right\|=0$.

Lemma $2.5(\mathrm{Xu}[14])$. Assume that $\left\{\alpha_{n}\right\}$ is a sequence of non-negative real numbers such that $\alpha_{n+1} \leq\left(1-\gamma_{n}\right) \alpha_{n}+\delta_{n}$, where $\gamma_{n}$ is a sequence in $(0,1)$ and $\delta_{n}$ is a sequence in $\mathbb{R}$ such that

(i) $\sum_{n=1}^{\infty} \gamma_{n}=\infty$,

(ii) $\lim \sup _{n \rightarrow \infty} \frac{\delta_{n}}{\gamma_{n}} \leq 0$ or $\sum_{n=1}^{\infty}\left|\delta_{n}\right|<\infty$

Then $\lim _{n \rightarrow \infty} \alpha_{n}=0$.

Lemma 2.6 ([23] Demiclosedness Principle). Let $H$ be a Hilbert space, $K$ a closed convex subset of $H$, and $T: K \rightarrow K$ a non-expansive mapping with $\operatorname{Fix}(T) \neq \emptyset$. If $\left\{x_{n}\right\}$ is a sequence in $K$ weakly converging to $x$ and if $\left\{(I-T) x_{n}\right\}$ converges strongly to $y$, then $(I-T) x=y$.

Lemma 2.7 Let $F$ be a $L$-Lipschitzian and $\eta$-strongly monotone operator on a nonempty closed convex subset $C$ of a real Hilbert space $H$ with $0<\eta \leq L$ and $0<t<2 \eta /$ $L^{2}$. Then $S=(I-t F): C \rightarrow C$ is a contraction with contraction coefficient $\tau_{t}=1-t\left(\eta-\frac{t L^{2}}{2}\right)$.

Proof. From the definition of $\eta$-strongly monotone and $L$-Lipschitzian operator, we have

$$
\begin{aligned}
\|S x-S y\|^{2} & \leq\|x-y-t(F x-F y)\|^{2} \\
& \leq\|x-y\|^{2}+t^{2}\|F x-F y\|^{2}-2 t\langle F x-F y, x-y\rangle \\
& \leq\|x-y\|^{2}+t^{2} L^{2}\|x-y\|^{2}-2 t \eta\|x-y\|^{2} \\
& =\left[1-t\left(2 \eta-t L^{2}\right)\right]\|x-y\|^{2} \\
& \leq\left[1-t\left(\eta-\frac{t L^{2}}{2}\right)\right]^{2}\|x-y\|^{2} .
\end{aligned}
$$


for all $x, y \in C$. From $0<\eta \leq L$ and $0<t<2 \eta / L^{2}$, we have $0<1-t\left(\eta-\frac{t L^{2}}{2}\right)<1$ and

$$
\|S x-S y\| \leq \tau_{t}\|x-y\|
$$

where $\tau_{t}=1-t\left(\eta-\frac{t L^{2}}{2}\right) \in(0,1)$. Hence, $S$ is a contraction with contraction coefficient $\tau_{t}$.

Lemma 2.8 ([23] Lemma 2.3). Let $\varphi$ be an MKC on a convex subset $C$ of a Banach space $E$. Then for each $\varepsilon>0$, there exists $r \in(0,1)$ such that $\|x-y\| \geq \varepsilon$ implies $\| \varphi x-$ $\varphi y\|\leq r\| x-y \|$ for all $x, y \in C$.

Lemma 2.9. Let $C$ be a closed convex subset of a Hilbert space $H$. Let $S: C \rightarrow C$ be a non-expansive mapping and $\varphi$ be an MKC on $C$. Suppose $F$ : $C \rightarrow C$ be a $\eta$-strongly monotone and $L$-Lipschitzian mapping with coefficient $\eta$ and $\eta>\gamma>0$. Then the sequence $\left\{x_{t}\right\}$ define by

$$
x_{t}=t \gamma \phi\left(x_{t}\right)+(1-t F) S x_{t}
$$

converges strongly as $t \rightarrow 0$ to a fixed point $\tilde{x}$ of $S$ which solves the variational inequality:

$$
\langle(F-\gamma \phi) \tilde{x}, \tilde{x}-z\rangle \leq 0, \quad \forall z \in F(S) .
$$

Proof. The definition of $\left\{x_{t}\right\}$ is well definition. Indeed, From the definition of MKC, we can see $\mathrm{MKC}$ is also a non-expansive mapping. Consider a mapping $S_{t}$ on $C$ defined by

$$
S_{t} x=t \gamma \phi(x)+(I-t F) S x, x \in C .
$$

It is easy to see that $S_{t}$ is a contraction, when $0<t<\frac{2(\eta-\gamma)}{L^{2}}$ Indeed, by Lemmas 2.7 and 2.8 , we have

$$
\begin{aligned}
\left\|S_{t} x-S_{t} y\right\| & \leq t \gamma\|\phi(x)-\phi(\gamma)\|+\|(I-t F) S x-(I-t F) S y\| \\
& \leq t \gamma\|\phi(x)-\phi(\gamma)\|+\tau_{t}\|S x-S \gamma\| \\
& \leq t \gamma\|x-\gamma\|+\tau_{t}\|x-\gamma\| \\
& \leq \theta_{t}\|x-\gamma\| .
\end{aligned}
$$

where $\theta_{t}=t \gamma+\tau_{t} \in(0,1)$. Hence, $S_{t}$ has a unique fixed point, denoted by $x_{t}$, which uniquely solves the fixed point equation

$$
x_{t}=t \gamma \phi\left(x_{t}\right)+(I-t F) S x_{t} .
$$

We next show the uniqueness of a solution of the variational inequality (2.1). Suppose both $\tilde{x} \in F(S)$ and $\hat{x} \in F(S)$ are solutions to (2.1). Not lost generality, we may assume there is a positive number $\varepsilon$ such that $\|\hat{x}-\tilde{x}\| \geq \varepsilon$. Then, by Lemma 2.8 , there is a number $r \in(0,1)$ such that $\|\phi \hat{x}-\phi \tilde{x}\| \leq r\|\hat{x}-\tilde{x}\|$. From (2.1), we know

$$
\langle(F-\gamma \phi) \tilde{x}, \tilde{x}-\hat{x}\rangle \leq 0 .
$$

and

$$
\langle(F-\gamma \phi) \hat{x}, \hat{x}-\tilde{x}\rangle \leq 0 .
$$


Adding up (2.2) and (2.3) gets

$$
\langle(F-\gamma \phi) \hat{x}-(F-\gamma \phi) \tilde{x}, \hat{x}-\tilde{x}\rangle \leq 0 .
$$

Noticing that

$$
\begin{aligned}
\langle(F-\gamma \phi) \hat{x}-(F-\gamma \phi) \tilde{x}, \hat{x}-\tilde{x}\rangle & =\langle F \hat{x}-F \tilde{x}, \hat{x}-\tilde{x}\rangle-\gamma\langle\phi \hat{x}-\phi \tilde{x}, \hat{x}-\tilde{x}\rangle \\
& \geq \eta\|\hat{x}-\tilde{x}\|^{2}-\gamma\|\phi \hat{x}-\phi \tilde{x}\|\|\hat{x}-\tilde{x}\| \\
& \geq \eta\|\hat{x}-\tilde{x}\|^{2}-\gamma r\|\hat{x}-\tilde{x}\|^{2} \\
& \geq(\eta-\gamma r)\|\hat{x}-\tilde{x}\|^{2} \\
& \geq(\eta-\gamma r) \varepsilon^{2} \\
& >0 .
\end{aligned}
$$

Therefore, $\hat{x}=\tilde{x}$ and the uniqueness is proved. Below we use $\tilde{x}$ to denote the unique solution of (2.1).

We observe that $\left\{x_{t}\right\}$ is bounded. Indeed, We may assume $0<t<\frac{\eta-\gamma}{L^{2}}$. For $\forall p \in F$ $(S)$, fixed $\varepsilon_{1}$, for each $t$

Case 1. $\left\|x_{t}-p\right\|<\varepsilon_{1}$; In this case, we can see easily that $\left\{x_{t}\right\}$ is bounded.

Case 2. $\left\|x_{t}-p\right\| \geq \varepsilon_{1}$. In this case, by Lemma 2.8, there is a number $r_{1} \in(0,1)$ such that

$$
\begin{aligned}
\| \phi\left(x_{t}\right)- & \phi(p)\left\|\leq r_{1}\right\| x_{t}-p \| . \\
\left\|x_{t}-p\right\| & =\left\|t \gamma \phi\left(x_{t}\right)+(I-t F) S x_{t}-p\right\| \\
& =\left\|t\left(\gamma \phi\left(x_{t}\right)-F p\right)+(I-t F) S x_{t}-(I-t F) p\right\| \\
& \leq t\left\|\gamma \phi\left(x_{t}\right)-F p\right\|+\tau_{t}\left\|x_{t}-p\right\| \\
& \leq t\left\|\gamma \phi\left(x_{t}\right)-\gamma \phi(p)\right\|+t\|\gamma \phi(p)-F p\|+\tau_{t}\left\|x_{t}-p\right\| \\
& \leq t \gamma r_{1}\left\|x_{t}-p\right\|+t\|\gamma \phi(p)-F p\|+\tau_{t}\left\|x_{t}-p\right\|
\end{aligned}
$$

therefore, $\left\|x_{t}-p\right\| \leq \frac{2\|\gamma \phi(p)-F p\|}{\eta-\gamma}$. This implies the $\left\{x_{t}\right\}$ is bounded.

Next, we prove that $x_{t} \rightarrow \tilde{x}$ as $t \rightarrow 0$.

Since $\left\{x_{t}\right\}$ is bounded and $H$ is reflexive, there exists a subsequence $\left\{x_{t_{n}}\right\}$ of $\left\{x_{t}\right\}$ such that $x_{t_{n}} \rightarrow x *$. By $x_{t}-S x_{t}=t\left(\gamma \varphi\left(x_{t}\right)-F S x_{t}\right)$, we have $x_{t_{n}}-S x_{t_{n}} \rightarrow 0$, as $t_{n} \rightarrow 0$. It follows from Lemma 2.6 that $x^{*} \in F(S)$.

We claim

$$
\left\|x_{t_{n}}-x^{*}\right\| \rightarrow 0
$$

By contradiction, there is a number $\varepsilon_{0}$ and a subsequence $\left\{x_{t_{m}}\right\}$ of $\left\{x_{t_{n}}\right\}$ such that $\left\|x_{t_{m}}-x^{*}\right\| \geq \varepsilon_{0}$. From Lemma 2.8, there is a number $r_{\varepsilon_{0}}>0$ such that $\left\|\phi\left(x_{t_{m}}\right)-\phi\left(x^{*}\right)\right\| \leq r_{\varepsilon_{0}}\left\|x_{t_{m}}-x^{*}\right\|$, we write

$$
x_{t_{m}}-x^{*}=t_{m}\left(\gamma \phi\left(x_{t_{m}}\right)-F x^{*}\right)+\left(I-t_{m} F\right) S x_{t_{m}}-\left(I-t_{m} F\right) x *,
$$

to derive that

$$
\begin{aligned}
\left\|x_{t_{m}}-x^{*}\right\|^{2} & =t_{m}\left\langle\gamma \phi\left(x_{t_{m}}\right)-F x^{*}, x_{t_{m}}-x^{*}\right\rangle+\left\langle\left(I-t_{m} F\right) S x_{t_{m}}-\left(I-t_{m} F\right) x^{*}, x_{t_{m}}-x^{*}\right\rangle \\
& \leq t_{m}\left\langle\gamma \phi\left(x_{t_{m}}\right)-F x^{*}, x_{t_{m}}-x^{*}\right\rangle+\tau_{t_{m}}\left\|x_{t_{m}}-x^{*}\right\|^{2} .
\end{aligned}
$$


It follows that

$$
\begin{aligned}
\left\|x_{t_{m}}-x^{*}\right\|^{2} & \leq\left(\eta-\frac{t_{m} L^{2}}{2}\right)^{-1}\left\langle\gamma \phi\left(x_{t_{m}}\right)-F x^{*}, x_{t_{m}}-x^{*}\right\rangle \\
& =\left(\eta-\frac{t_{m} L^{2}}{2}\right)^{-1}\left[\left\langle\gamma \phi\left(x_{t_{m}}\right)-\gamma \phi\left(x^{*}\right), x_{t_{m}}-x^{*}\right\rangle+\left\langle\gamma \phi\left(x^{*}\right)-F x^{*}, x_{t_{m}}-x^{*}\right\rangle\right] \\
& \leq\left(\eta-\frac{t_{m} L^{2}}{2}\right)^{-1}\left[\gamma r_{\varepsilon_{0}}\left\|x_{t_{m}}-x^{*}\right\|^{2}+\left\langle\gamma \phi\left(x^{*}\right)-F x^{*}, x_{t_{m}}-x^{*}\right\rangle\right] .
\end{aligned}
$$

Therefore,

$$
\left\|x_{t_{m}}-x^{*}\right\|^{2} \leq \frac{1}{\eta-\gamma \varepsilon_{0}-\frac{t_{m} L^{2}}{2}}\left\langle\gamma \phi\left(x^{*}\right)-F x^{*}, x_{t_{m}}-x^{*}\right\rangle .
$$

By (2.5), we get that $x_{t_{m}} \rightarrow x^{*}$. It is a contradiction. Hence, we have $x_{t_{n}} \rightarrow x^{*}$.

We next prove that $x^{*}$ solves the variational inequality (2.1). Since

$$
x_{t}=t \gamma \phi\left(x_{t}\right)+(I-t F) S x_{t}
$$

we derive that

$$
(F-\gamma \phi) x_{t}=-\frac{1}{t}\left[(I-S) x_{t}-t\left(F x_{t}-F S x_{t}\right)\right] .
$$

Notice

$$
\begin{aligned}
\left\langle(I-S) x_{t}-(I-S) z, x_{t}-z\right\rangle & \geq\left\|x_{t}-z\right\|^{2}-\left\|S x_{t}-S z\right\|\left\|x_{t}-z\right\| \\
& \geq\left\|x_{t}-z\right\|^{2}-\left\|x_{t}-z\right\|^{2} \\
& =0 .
\end{aligned}
$$

It follows that, for $\forall z \in F(S)$,

$$
\begin{aligned}
\left\langle(F-\gamma \phi) x_{t}, x_{t}-z\right\rangle & =-\frac{1}{t}\left\langle(I-S) x_{t}-t\left(F x_{t}-F S x_{t}\right), x_{t}-z\right\rangle \\
& =-\frac{1}{t}\left\langle(I-S) x_{t}-(I-S) z, x_{t}-z\right\rangle+\left\langle F x_{t}-F\left(S x_{t}\right), x_{t}-z\right\rangle \\
& \leq L\left\|x_{t}-S x_{t}\right\|\left\|x_{t}-z\right\| .
\end{aligned}
$$

Noticing

$$
x_{t}-S x_{t}=t\left[\gamma \phi\left(x_{t}\right)-F S x_{t}\right] .
$$

Hence, we have

$$
x_{t}-S x_{t} \rightarrow 0, \quad \text { as } t \rightarrow 0 .
$$

Now replacing $t$ in (2.6) with $t_{n}$ and letting $n \rightarrow \infty$, noticing $(I-S) x_{t_{n}} \rightarrow(I-S) x^{*}=0$ for $x^{*} \in F(S)$, we obtain $\left\langle(F-\gamma \varphi) x^{*}, x^{*}-z\right\rangle \leq 0$. That is, $x^{*} \in$ $F(S)$ is a solution of (2.1); Hence, $\tilde{x}=x^{*}$ by uniqueness. We have shown that each cluster point of $x_{t}$ (at $t \rightarrow 0$ ) equals $\tilde{x}$. Therefore, $x_{t} \rightarrow \tilde{x}$ as $t \rightarrow 0$.

Lemma 2.10. Let $H$ be a Hilbert space and $C$ be a nonempty convex subset of $H$. Assume that $T_{i}: C \rightarrow E$ is a countable family of $k_{i}$-strict pseudo-contraction for some $0 \leq k_{i}<1$ and $\sup \left\{k_{i}: i \in \mathbb{N}\right\}<1$ such that $\bigcap_{i=1}^{\infty} F\left(T_{i}\right) \neq \emptyset$. Assume that $\left\{\mu_{i}\right\}$ is a positive sequence such that $\sum_{i=1}^{\infty} \mu_{i}=1$. Then $\sum_{i=1}^{\infty} \mu_{i} T_{i}: C \rightarrow E$ is a $k$-strict pseudo-contraction with $k=\sup \left\{k_{i}: i \in \mathbb{N}\right\}$ and $F\left(\sum_{i=1}^{\infty} \mu_{i} T_{i}\right)=\bigcap_{i=1}^{\infty} F\left(T_{i}\right)$. 
Proof. Let

$$
G_{n} x=\mu_{1} T_{1} x+\mu_{2} T_{2} x+\cdots+\mu_{n} T_{n} x
$$

and $\sum_{i=1}^{n} \mu_{i}=1$. Then, $G_{n}: C \rightarrow E$ is a $k_{i}$-strict pseudo-contraction with $k=\max \left\{k_{i}\right.$ : $1 \leq i \leq n\}$. Indeed, we can firstly see the case of $n=2$.

$$
\begin{aligned}
& \left\langle\left(I-G_{2}\right) x-\left(I-G_{2}\right) y, x-\gamma\right\rangle \\
= & \left.\left.\left\langle\mu_{1}\left(I-T_{1}\right) x+\mu_{2}\left(I-T_{2}\right)\right) x-\mu_{1}\left(I-T_{1}\right) y-\mu_{2}\left(I-T_{2}\right)\right) y, x-\gamma\right\rangle \\
= & \mu_{1}\left\langle\left(I-T_{1}\right) x-\left(I-T_{1}\right) y, x-\gamma\right\rangle+\mu_{2}\left\langle\left(I-T_{2}\right) x-\left(I-T_{2}\right) y, x-\gamma\right\rangle \\
\geq & \mu_{1} \frac{1-k_{1}}{2}\left\|\left(I-T_{1}\right) x-\left(I-T_{1}\right) y\right\|^{2}+\mu_{2} \frac{1-k_{2}}{2}\left\|\left(I-T_{2}\right) x-\left(I-T_{2}\right) y\right\|^{2} \\
\geq & \frac{1-k}{2}\left[\mu_{1}\left\|\left(I-T_{1}\right) x-\left(I-T_{1}\right) y\right\|^{2}+\mu_{2}\left\|\left(I-T_{2}\right) x-\left(I-T_{2}\right) y\right\|^{2}\right] \\
\geq & \frac{1-k}{2}\left\|\left(I-G_{2}\right) x-\left(I-G_{2}\right) y\right\|^{2},
\end{aligned}
$$

which shows that $G_{2}: C \rightarrow E$ is a $k$-strict pseudo-contraction with $k=\max \left\{k_{i}: i=1,2\right\}$. By the same way, our proof method easily carries over to the general finite case.

Next, we prove the infinite case. From the definition of $k$-strict pseudo-contraction, we know

$$
\left\langle\left(I-T_{n}\right) x-\left(I-T_{n}\right) y, x-y\right\rangle \geq \frac{1-k}{2}\left\|\left(I-T_{n}\right) x-\left(I-T_{n}\right) y\right\|^{2} .
$$

Hence, we can get

$$
\left\|\left(I-T_{n}\right) x-\left(I-T_{n}\right) y\right\| \leq \frac{2}{1-k}\|x-y\| .
$$

Taking $p \in F\left(T_{n}\right)$, from 2.7 we have

$$
\left\|\left(I-T_{n}\right) x\right\|=\left\|\left(I-T_{n}\right) x-\left(I-T_{n}\right) p\right\| \leq \frac{2}{1-k}\|x-p\| .
$$

Consequently, for $\forall x \in H$, if $\bigcap_{i=1}^{\infty} F\left(T_{i}\right) \neq \emptyset, \mu_{i}>0$ and $\sum_{i=1}^{\infty} \mu_{i}=1$, then $\sum_{i=1}^{\infty} \mu_{i} T_{i}$ strongly converges.

Let

$$
T x=\sum_{i=1}^{\infty} \mu_{i} T_{i} x
$$

we have

$$
T x=\sum_{i=1}^{\infty} \mu_{i} T_{i} x=\lim _{n \rightarrow \infty} \sum_{i=1}^{n} \mu_{i} T_{i} x=\lim _{n \rightarrow \infty} \frac{1}{\sum_{i=1}^{n} \mu_{i}} \sum_{i=1}^{n} \mu_{i} T_{i} x .
$$

Hence,

$$
\begin{aligned}
& \langle(I-T) x-(I-T) y, x-\gamma\rangle \\
= & \lim _{n \rightarrow \infty}\left\langle\left(I-\frac{1}{\sum_{i=1}^{n} \mu_{i}} \sum_{i=1}^{n} \mu_{i} T_{i}\right) x+\left(I-\frac{1}{\sum_{i=1}^{n} \mu_{i}} \sum_{i=1}^{n} \mu_{i} T_{i}\right) y, x-\gamma\right\rangle \\
= & \lim _{n \rightarrow \infty} \frac{1}{\sum_{i=1}^{n} \mu_{i}} \sum_{i=1}^{n} \mu_{i}\left\langle\left(I-T_{i}\right) x-\left(I-T_{i}\right) y, x-\gamma\right\rangle \\
\geq & \lim _{n \rightarrow \infty} \frac{1}{\sum_{i=1}^{n} \mu_{i}} \sum_{i=1}^{n} \mu_{i} \frac{1-k}{2}\left\|\left(I-T_{i}\right) x-\left(I-T_{i}\right) \gamma\right\|^{2} \\
\geq & \frac{1-k}{2} \lim _{n \rightarrow \infty}\left\|\left(I-\frac{1}{\sum_{i=1}^{n} \mu_{i}} \sum_{i=1}^{n} \mu_{i} T_{i}\right) x-\left(I-\frac{1}{\sum_{i=1}^{n} \mu_{i}} \sum_{i=1}^{n} \mu_{i} T_{i}\right) \gamma\right\|^{2} \\
= & \frac{1-k}{2}\|(I-T) x-(I-T) \gamma\|^{2} .
\end{aligned}
$$


So, we get $T$ is $k$-strict pseudo-contraction.

Finally, we show $F\left(\sum_{i=1}^{\infty} \mu_{i} T_{i}\right)=\bigcap_{i=1}^{\infty} F\left(T_{i}\right)$. Suppose that $x=\sum_{i=1}^{\infty} \mu_{i} T_{i} x$, it is sufficient to show that $x \in \bigcap_{i=1}^{\infty} F\left(T_{i}\right)$. Indeed, for $p \in \bigcap_{i=1}^{\infty} F\left(T_{i}\right)$, we have

$$
\begin{aligned}
\|x-p\|^{2} & =\langle x-p, x-p\rangle \\
& =\left\langle\sum_{i=1}^{\infty} \mu_{i} T_{i} x-p, x-p\right\rangle \\
& =\sum_{i=1}^{\infty} \mu_{i}\left\langle T_{i} x-p, x-p\right\rangle \\
& \leq\|x-p\|^{2}-\frac{1-k}{2} \sum_{i=1}^{\infty} \mu_{i}\left\|x-T_{i} x\right\|^{2},
\end{aligned}
$$

where $k=\sup \left\{k_{i}: i \in \mathbb{N}\right\}$. Hence, we get $x=T_{i} x$, this means that $x \in \bigcap_{i=1}^{\infty} F\left(T_{i}\right)$.

\section{Main results}

Lemma 3.1. Let $C$ be a closed convex subset of a real Hilbert space $E$ such that $C+C$ $\subset C$. Let $\varphi$ be a MKC on $C$. Suppose $F: C \rightarrow C$ be a $\eta$-strongly monotone and $L$-Lipschitzian operator and $0<\gamma<\eta$ and $T_{i}: C \rightarrow E$ be $k_{i}$-strictly pseudo-contractive non-self-mapping such that $\bigcap_{i=1}^{\infty} F\left(T_{i}\right) \neq \emptyset$. Assume $k=\sup \left\{k_{i}: i \in \mathbb{N}\right\}<1$. Let $\left\{x_{n}\right\}$ be a sequence of $C$ generated by (1.5) with the sequences $\left\{\alpha_{n}\right\},\left\{\beta_{n}\right\}$ and $\left\{\gamma_{n}\right\}$ in $[0,1]$, assume for each $n,\left\{\mu_{i}^{(n)}\right\}$ is a infinity sequence of positive number such that $\sum_{i=1}^{\infty} \mu_{i}^{(n)}=1$.

The following control conditions are satisfied

(i) $\sum_{i=1}^{\infty} \alpha_{n}=\infty, \quad \lim _{n \rightarrow \infty} \alpha_{n}=0$

(ii) $k \leq \beta_{n}<1$,

(iii) $\lim _{n \rightarrow \infty}\left(\beta_{n+1}-\beta_{n}\right)=0, \quad \lim _{n \rightarrow \infty} \sum_{i=1}^{\infty}\left|\mu_{i}^{(n+1)}-\mu_{i}^{(n)}\right|=0$

(iv) $0<\liminf _{n \rightarrow \infty} \gamma_{n} \leq \limsup _{n \rightarrow \infty} \gamma_{n}<1$.

Then $\lim _{n \rightarrow \infty}\left\|x_{n+1}-x_{n}\right\|=0$.

Proof. Write, for each $n \geq 0, B_{n}=\sum_{i=1}^{\infty} \mu_{i}^{(n)} T_{i}$. By Lemma 2.10, each $B_{n}$ is a $k$-strict pseudo-contraction on $C$ and $F\left(B_{n}\right)=\bigcap_{i=1}^{\infty} F\left(T_{i}\right)$ for all $n$ and the algorithm (1.5) can be rewritten as

$$
\left\{\begin{array}{l}
x_{1}=x \in C, \\
y_{n}=P_{C}\left[\beta_{n} x_{n}+\left(1-\beta_{n}\right) B_{n} x_{n}\right] \\
x_{n+1}=\alpha_{n} \gamma \phi\left(x_{n}\right)+\gamma_{n} x_{n}+\left(\left(1-\gamma_{n}\right) I-\alpha_{n} F\right) y_{n}, n \geq 1 .
\end{array},\right.
$$

The rest of the proof will now be split into two parts.

Step 1. First, we show that sequences $\left\{x_{n}\right\}$ and $\left\{y_{n}\right\}$ are bounded. Define a mapping

$$
L_{n} x: P_{C}\left[\beta_{n} x+\left(1-\beta_{n}\right) B_{n} x\right] .
$$


Then from the control condition (ii), Lemma 2.3, we obtain $L_{n}: C \rightarrow C$ is nonexpansive. Taking a point $p \in \bigcap_{i=1}^{\infty} F\left(T_{i}\right)$, by Lemma 2.2 , we can get $L_{n} p=p$. Hence, we have

$$
\left\|y_{n}-p\right\|=\left\|L_{n} x_{n}-p\right\| \leq\left\|x_{n}-p\right\| .
$$

Not lose generality, we can assume $r_{n} \leq b<1$, and $0<\alpha_{n}<\frac{(\eta-\gamma)(1-b)}{L^{2}}$. From definition of MKC and Lemma 2.8, for any $\varepsilon>0$ there is a number $r_{\varepsilon} \in(0,1)$, if $\| x_{t}$ $p \|<\varepsilon$ then $\left\|\varphi\left(x_{t}\right)-\varphi(p)\right\|<\varepsilon$; If $\left\|x_{t}-p\right\| \geq \varepsilon$ then $\left\|\varphi\left(x_{t}\right)-\varphi(p)\right\| \leq r_{\varepsilon}\left\|x_{t}-p\right\|$. It follows 3.1 and Lemma 2.7 that

$$
\begin{aligned}
\left\|x_{n+1}-p\right\|= & \left\|\alpha_{n} \gamma \phi\left(x_{n}\right)+\gamma_{n} x_{n}+\left(\left(1-\gamma_{n}\right) I-\alpha_{n} F\right) \gamma_{n}-p\right\| \\
= & \left\|\alpha_{n}\left(\gamma \phi\left(x_{n}\right)-F p\right)+\gamma_{n}\left(x_{n}-p\right)+\left[\left(1-\gamma_{n}\right) I-\alpha_{n} F\right] y_{n}-\left[\left(1-\gamma_{n}\right) I-\alpha_{n} F\right] p\right\| \\
\leq & {\left[1-\gamma_{n}-\alpha_{n}\left(\eta-\frac{\alpha_{n} L^{2}}{2\left(1-\gamma_{n}\right)}\right)\right]\left\|x_{n}-p\right\|+\gamma_{n}\left\|x_{n}-p\right\|+\alpha_{n}\left\|\gamma \phi\left(x_{n}\right)-F p\right\| } \\
\leq & {\left.\left[1-\alpha_{n}\left(\eta-\frac{\alpha_{n} L^{2}}{2\left(1-\gamma_{n}\right)}\right)\right]\left\|x_{n}-p\right\|+\alpha_{n} \gamma \max \left\{r_{\varepsilon}\left\|x_{n}-p\right\|, \varepsilon\right\}+\alpha_{n}\|\gamma \phi(p)-F p\|\right\} } \\
= & \max \left\{\left[1-\alpha_{n}\left(\eta-\frac{\alpha_{n} L^{2}}{2\left(1-\gamma_{n}\right)}\right)\right]\left\|x_{n}-p\right\|+\alpha_{n} \gamma r_{\varepsilon}\left\|x_{n}-p\right\|+\alpha_{n}\|\gamma \phi(p)-F p\|,\right. \\
& {\left.\left[1-\alpha_{n}\left(\eta-\frac{\alpha_{n} L^{2}}{2\left(1-\gamma_{n}\right)}\right)\right]\left\|x_{n}-p\right\|+\alpha_{n} \gamma \varepsilon+\alpha_{n}\|\gamma \phi(p)-F p\|\right\} } \\
= & \max \left\{\left[1-\alpha_{n}\left(\eta-\frac{\alpha_{n} L^{2}}{2\left(1-\gamma_{n}\right)}-\gamma r_{\varepsilon}\right)\right]\left\|x_{n}-p\right\|+\alpha_{n}\|\gamma \phi(p)-F p\|,\right. \\
& {\left.\left[1-\alpha_{n}\left(\eta-\frac{\alpha_{n} L^{2}}{2\left(1-\gamma_{n}\right)}\right)\right]\left\|x_{n}-p\right\|+\alpha_{n} \gamma \varepsilon+\alpha_{n}\|\gamma \phi(p)-F p\|\right\} . }
\end{aligned}
$$

By induction, we have

$$
\begin{aligned}
& \left\|x_{n+1}-p\right\| \\
\leq & \max \left\{\left[1-\alpha_{n}\left(\eta-\frac{\alpha_{n} L^{2}}{2\left(1-\gamma_{n}\right)}-\gamma r_{\varepsilon}\right)\right]\left\|x_{n}-p\right\|+\alpha_{n}\left(\eta-\frac{\alpha_{n} L^{2}}{2\left(1-\gamma_{n}\right)}-\gamma r_{\varepsilon}\right) \frac{\|\gamma \phi(p)-F p\|}{\eta-\frac{\alpha_{n} L^{2}}{2\left(1-\gamma_{n}\right)}-\gamma r_{\varepsilon}},\right. \\
& {\left.\left[1-\alpha_{n}\left(\eta-\frac{\alpha_{n} L^{2}}{2\left(1-\gamma_{n}\right)}\right)\right]\left\|x_{n}-p\right\|+\alpha_{n}\left[\eta-\frac{\alpha_{n} L^{2}}{2\left(1-\gamma_{n}\right)}\right] \frac{\gamma \varepsilon+\|\gamma \phi(p)-F p\|}{\eta-\frac{\alpha_{n} L^{2}}{2(1-\gamma n)}}\right\} } \\
\leq & \max \left\{\left[1-\alpha_{n}\left(\eta-\frac{\alpha_{n} L^{2}}{2\left(1-\gamma_{n}\right)}-\gamma r_{\varepsilon}\right)\right]\left\|x_{n}-p\right\|+\alpha_{n}\left[\eta-\frac{\alpha_{n} L^{2}}{2\left(1-\gamma_{n}\right)}-\gamma r_{\varepsilon}\right] \frac{2\|\gamma \phi(p)-F p\|}{\eta-\gamma},\right. \\
& {\left.\left[1-\alpha_{n}\left(\eta-\frac{\alpha_{n} L^{2}}{2\left(1-\gamma_{n}\right)}\right)\right]\left\|x_{n}-p\right\|+\alpha_{n}\left[\eta-\frac{\alpha_{n} L^{2}}{2\left(1-\gamma_{n}\right)}\right] \frac{2(\gamma \varepsilon+\|\gamma \phi(p)-F p\|)}{\eta-\gamma}\right\} . }
\end{aligned}
$$

Hence, we have

$$
\left\|x_{n}-p\right\| \leq \max \left\{\left\|x_{0}-p\right\|, \frac{2(\gamma \varepsilon+\|\gamma \phi(p)-F p\|)}{\eta+\gamma r_{\varepsilon}}\right\}, \quad n \geq 0,
$$

which gives that the sequence $\left\{x_{n}\right\}$ is bounded, so are $\left\{y_{n}\right\}$ and $\left\{L_{n} x_{n}\right\}$.

Step 2. In this part, we shall claim that $\left\|x_{n+1}-x_{n}\right\| \rightarrow 0$, as $n \rightarrow \infty$. From(3.1), we get

$$
x_{n+1}=\alpha_{n} \gamma \phi\left(x_{n}\right)+\gamma_{n} x_{n}+\left(\left(1-\gamma_{n}\right) I-\alpha_{n} F\right) L_{n} x_{n} .
$$

Define

$$
x_{n+1}=\left(1-\gamma_{n}\right) l_{n}+\gamma_{n} x_{n}, \quad \forall n \geq 0,
$$


where

$$
l_{n}=\frac{x_{n+1}-\gamma_{n} x_{n}}{1-\gamma_{n}} .
$$

It follows that

$$
\begin{aligned}
& l_{n+1}-l_{n} \\
= & \frac{\alpha_{n+1} \gamma \phi\left(x_{n+1}\right)+\gamma_{n+1} x_{n+1}+\left[\left(1-\gamma_{n+1}\right) I-\alpha_{n+1} F\right] L_{n+1} x_{n+1}-\gamma_{n+1} x_{n+1}}{1-\gamma_{n+1}} \\
& -\frac{\alpha_{n} \gamma \phi\left(x_{n}\right)+\gamma_{n} x_{n}+\left[\left(1-\gamma_{n}\right) I-\alpha_{n} F\right] L_{n} x_{n}-\gamma_{n} x_{n}}{1-\gamma_{n}} \\
= & \frac{\alpha_{n+1}\left[\gamma \phi\left(x_{n+1}\right)-F L_{n+1} x_{n+1}\right]}{1-\gamma_{n+1}}-\frac{\alpha_{n}\left[\gamma \phi\left(x_{n}\right)-F L_{n} x_{n}\right]}{1-\gamma_{n}}+L_{n+1} x_{n+1}-L_{n} x_{n}
\end{aligned}
$$

which yields that

$$
\begin{aligned}
& \left\|l_{n+1}-l_{n}\right\| \\
\leq & \frac{\alpha_{n+1}\left\|\gamma \phi\left(x_{n+1}\right)-F L_{n+1} x_{n+1}\right\|}{1-\gamma_{n+1}}+\frac{\alpha_{n}\left\|\gamma \phi\left(x_{n}\right)-F L_{n} x_{n}\right\|}{1-\gamma_{n}}+\left\|L_{n+1} x_{n+1}-L_{n} x_{n}\right\| \\
\leq & \frac{\alpha_{n+1}\left\|\gamma \phi\left(x_{n+1}\right)-F L_{n+1} x_{n+1}\right\|}{1-\gamma_{n+1}}+\frac{\alpha_{n}\left\|\gamma \phi\left(x_{n}\right)-F L_{n} x_{n}\right\|}{1-\gamma_{n}}+\left\|L_{n+1} x_{n+1}-L_{n+1} x_{n}\right\| \\
& +\left\|L_{n+1} x_{n}-L_{n} x_{n}\right\| \\
\leq & \frac{\alpha_{n+1}\left\|\gamma \phi\left(x_{n+1}\right)-F L_{n+1} x_{n+1}\right\|}{1-\gamma_{n+1}}+\frac{\alpha_{n}\left\|\gamma \phi\left(x_{n}\right)-F L_{n} x_{n}\right\|}{1-\gamma_{n}}+\left\|x_{n+1}-x_{n}\right\| \\
& +\left\|L_{n+1} x_{n}-L_{n} x_{n}\right\| .
\end{aligned}
$$

Next, we estimate $\left\|L_{n+1} x_{n}-L_{n} x_{n}\right\|$. Notice that

$$
\begin{aligned}
\left\|L_{n+1} x_{n}-L_{n} x_{n}\right\| & =\left\|P_{C}\left[\beta_{n+1} x_{n}+\left(1-\beta_{n+1}\right) B_{n+1} x_{n}\right]-P_{C}\left[\beta_{n} x_{n}+\left(1-\beta_{n}\right) B_{n} x_{n}\right]\right\| \\
& \leq\left\|\left[\beta_{n+1} x_{n}+\left(1-\beta_{n+1}\right) B_{n+1} x_{n}\right]-\left[\beta_{n} x_{n}+\left(1-\beta_{n}\right) B_{n} x_{n}\right]\right\| \\
& \leq\left\|x_{n}-B_{n+1} x_{n}\right\|\left|\beta_{n+1}-\beta_{n}\right|+\left(1-\beta_{n}\right)\left\|B_{n+1} x_{n}-B_{n} x_{n}\right\| \\
& \leq\left\|x_{n}-B_{n+1} x_{n}\right\|\left|\beta_{n+1}-\beta_{n}\right|+\left(1-\beta_{n}\right) \sum_{i=1}^{\infty}\left|\mu_{i}^{(n+1)}-\mu_{i}^{(n)}\right|\left\|T_{i} x_{n}\right\| .
\end{aligned}
$$

Substituting (3.5) into (3.4), we have

$$
\begin{aligned}
\left\|l_{n+1}-l_{n}\right\| \leq & \frac{\alpha_{n+1}\left\|\gamma \phi\left(x_{n+1}\right)-F L_{n+1} x_{n+1}\right\|}{1-\gamma_{n+1}}+\frac{\alpha_{n}\left\|\gamma \phi\left(x_{n}\right)-F L_{n} x_{n}\right\|}{1-\gamma_{n}}+\left\|x_{n+1}-x_{n}\right\| \\
& +\left\|x_{n}-B_{n+1} x_{n}\right\|\left|\beta_{n+1}-\beta_{n}\right|+\left(1-\beta_{n}\right) \sum_{i=1}^{\infty}\left|\mu_{i}^{(n+1)}-\mu_{i}^{(n)}\right|\left\|T_{i} x_{n}\right\| .
\end{aligned}
$$

Hence, we have

$$
\begin{aligned}
& \left\|l_{n+1}-l_{n}\right\|-\left\|x_{n+1}-x_{n}\right\| \\
& \leq \frac{\alpha_{n+1}\left\|\gamma \phi\left(x_{n+1}\right)-F L_{n+1} x_{n+1}\right\|}{1-\gamma_{n+1}}+\frac{\alpha_{n}\left\|\gamma \phi\left(x_{n}\right)-F L_{n} x_{n}\right\|}{1-\gamma_{n}}+\left\|x_{n}-B_{n+1} x_{n}\right\|\left|\beta_{n+1}-\beta_{n}\right| \\
& \quad+\left(1-\beta_{n}\right) \sum_{i=1}^{\infty}\left|\mu_{i}^{(n+1)}-\mu_{i}^{(n)}\right|\left\|T_{i} x_{n}\right\| .
\end{aligned}
$$

Observing conditions (i), (iii), (iv), and the boundedness of $\left\{x_{n}\right\}$ it follows that

$$
\limsup _{n \rightarrow \infty}\left\{\left\|l_{n+1}-l_{n}\right\|-\left\|x_{n+1}-x_{n}\right\|\right\} \leq 0 .
$$


Thus by Lemma 2.4, we have $\lim _{n} \rightarrow \infty\left\|l_{n}-x_{n}\right\|=0$.

From (3.3), we have

$$
x_{n+1}-x_{n}=\left(1-\gamma_{n}\right)\left(l_{n}-x_{n}\right) .
$$

Therefore,

$$
\lim _{n \rightarrow \infty}\left\|x_{n+1}-x_{n}\right\|=0 .
$$

Theorem 3.2. Let $H$ be a real Hilbert space and $C$ is a closed convex subset of $H$ such that $C+C \subset C$. Let $\varphi$ be an MKC on $C$. Suppose $F: C \rightarrow C$ is $\eta$-strongly monotone and $L$-Lipschitzian operator and $\eta>\gamma>0$. Let $T_{i}: C \rightarrow E$ be $k_{i}$-strictly pseudocontractive non-self-mapping such that $\bigcap_{i=1}^{\infty} F\left(T_{i}\right) \neq \emptyset$. Assume $k=\sup \left\{k_{i}: i \in \mathbb{N}\right\}<1$. Let $\left\{x_{n}\right\}$ be a sequence of $C$ generated by (1.5) with the sequences $\left\{\alpha_{n}\right\},\left\{\beta_{n}\right\}$ and $\left\{\gamma_{n}\right\}$ in $[0,1]$. Assume for each $n, \sum_{i=1}^{\infty} \mu_{i}^{(n)}=1$ for all $n$ and $\mu_{i}^{(n)}>0$ for all $i \in \mathbb{N}$. They satisfy the conditions (i), (ii), (iii), (iv) of Lemma 3.1 and (v) $\lim _{n} \rightarrow \infty \beta_{n}=\alpha$, $\lim _{n \rightarrow \infty} \sum_{i=1}^{\infty}\left|\mu_{i}^{n}-\mu_{i}\right|=0$ and $\sum_{i=1}^{\infty} \mu_{i}=1\left(\mu_{i}>0\right)$. Then $\left\{x_{n}\right\}$ converges strongly to $\tilde{x} \in F$, which also solves the following variational inequality

$$
\langle\gamma \phi(\tilde{x})-F \tilde{x}, p-\tilde{x}\rangle \leq 0, \quad \forall p \in \bigcap_{i=1}^{\infty} F\left(T_{i}\right) .
$$

Proof. From (3.1), we obtain

$$
\begin{aligned}
\left\|L_{n} x_{n}-x_{n}\right\| & \leq\left\|x_{n}-x_{n+1}\right\|+\left\|x_{n+1}-L_{n} x_{n}\right\| \\
& =\left\|x_{n}-x_{n+1}\right\|+\left\|\alpha_{n} \gamma \phi\left(x_{n}\right)+\gamma_{n}\left(x_{n}-L_{n} x_{n}\right)-\alpha_{n} F L_{n} x_{n}\right\| \\
& \leq\left\|x_{n}-x_{n+1}\right\|+\alpha_{n}\left(\left\|\gamma \phi\left(x_{n}\right)\right\|+\left\|F L_{n} x_{n}\right\|\right)+\gamma_{n}\left\|x_{n}-L_{n} x_{n}\right\| .
\end{aligned}
$$

So $\left\|L_{n} x_{n}-x_{n}\right\| \leq \frac{1}{1-\gamma_{n}}\left(\left\|x_{n}-x_{n+1}\right\|+\alpha_{n}\left(\left\|\gamma \phi\left(x_{n}\right)\right\|+\left\|F L_{n} x_{n}\right\|\right)\right.$, which together with the condition (i), (iv) and Lemma 3.1 implies

$$
\lim _{n \rightarrow \infty}\left\|L_{n} x_{n}-x_{n}\right\|=0 .
$$

Define $B=\sum_{i=1}^{\infty} \mu_{i} T_{i}$, then $B: C \rightarrow \mathrm{E}$ is a $k$-strict pseudo-contraction such that $F(B)=\bigcap_{i=1}^{\infty} F\left(T_{i}\right)$ by Lemma 2.10, furthermore $B_{n} x \rightarrow B x$ as $n \rightarrow \infty$ for all $x \in C$ by (v). Defines $T: C \rightarrow E$ by

$$
T x=\alpha x+(1-\alpha) B x .
$$

Then, $T$ is non-expansive with $F(T)=F(B)$ by Lemma 2.3. It follows from Lemma 2.2 that $F\left(P_{C} T\right)=F(T)$. Notice that

$$
\begin{aligned}
\left\|P_{C} T x_{n}-x_{n}\right\| & \leq\left\|x_{n}-L_{n} x_{n}\right\|+\left\|L_{n} x_{n}-P_{C} T x_{n}\right\| \\
& \leq\left\|x_{n}-L_{n} x_{n}\right\|+\left\|\beta_{n} x_{n}+\left(1-\beta_{n}\right) B_{n} x_{n}-\left[\alpha x_{n}+(1-\alpha) B x_{n}\right]\right\| \\
& \leq\left\|x_{n}-L_{n} x_{n}\right\|+\left\|\left(\beta_{n}-\alpha\right)\left(x_{n}-B_{n} x_{n}\right)+(1-\alpha)\left(B_{n} x_{n}-B x_{n}\right)\right\| \\
& \leq\left\|x_{n}-L_{n} x_{n}\right\|+\left(\beta_{n}-a\right)\left\|x_{n}-B_{n} x_{n}\right\|+(1-\alpha)\left\|B_{n} x_{n}-B x_{n}\right\|
\end{aligned}
$$

which combines with (3.7) yielding that

$$
\lim _{n \rightarrow \infty}\left\|P_{C} T x_{n}-x_{n}\right\|=0 .
$$


Next, we show that

$$
\limsup _{n \rightarrow \infty}\left\langle\gamma \phi(\tilde{x})-F \tilde{x}, x_{n}-\tilde{x}\right\rangle \leq 0,
$$

where $\tilde{x}=\lim _{t \rightarrow 0} x_{t}$ with $x_{t}$ being the fixed point of the contraction

$$
x \mapsto t \gamma \phi(x)+(1-t F) P_{C} T x .
$$

To see this, we take a subsequence $\left\{x_{n_{k}}\right\}$ of $\left\{x_{n}\right\}$ such that

$$
\limsup _{n \rightarrow \infty}\left\langle\gamma \phi(\tilde{x})-F \tilde{x}, x_{n}-\tilde{x}\right\rangle=\lim _{k \rightarrow \infty}\left\langle\gamma \phi(\tilde{x})-F \tilde{x}, x_{n_{k}}-\tilde{x}\right\rangle .
$$

We may also assume that $x_{n_{k}} \rightarrow q$. Note that $q \in F(T)$ in virtue of Lemmas 2.6, 2.2, and (3.8). It follows from Lemma 2.9, we can get that

$$
\limsup _{n \rightarrow \infty}\left\langle\gamma \phi(\tilde{x})-F \tilde{x}, x_{n}-\tilde{x}\right\rangle=\lim _{k \rightarrow \infty}\left\langle\gamma \phi(\tilde{x})-F \tilde{x}, x_{n_{k}}-\tilde{x}\right\rangle=\langle\gamma \phi(\tilde{x})-F \tilde{x}, q-\tilde{x}\rangle \leq 0 .
$$

Finally, we show $\left\|x_{n}-\tilde{x}\right\| \rightarrow 0$. By contradiction, there is a number $\varepsilon_{0}$ such that

$$
\limsup _{n \rightarrow \infty}\left\|x_{n}-\tilde{x}\right\| \geq \varepsilon_{0} \text {. }
$$

Case 1. Fixed $\varepsilon_{1}\left(\varepsilon_{1}<\varepsilon_{0}\right)$, if for some $n \geq N \in \mathbb{N}$ such that $\left\|x_{n}-\tilde{x}\right\| \geq \varepsilon_{0}-\varepsilon_{1}$, and for the other $n \geq N \in \mathbb{N}$ such that $\left\|x_{n}-\tilde{x}\right\|<\varepsilon_{0}-\varepsilon_{1}$.

Let

$$
M_{n}=\frac{2\left\langle\gamma \phi(\tilde{x})-F \tilde{x}, x_{n+1}-\tilde{x}\right\rangle}{\left(\varepsilon_{0}-\varepsilon_{1}\right)^{2}} .
$$

From 3.9, we know $\lim \sup _{n \rightarrow \infty} M_{n} \leq 0$. Hence, there are two numbers $h$ and $N$, when $n>N$ we have $M_{n} \leq h$, where $h=\min \left\{\eta-\frac{\alpha_{n} L^{2}}{2\left(1-\gamma_{n}\right)}-\gamma\right\}$. From the above introduction, we can extract a number $n_{0}>N$ satisfying $\left\|x_{n_{0}}-\tilde{x}\right\|<\varepsilon_{0}-\varepsilon_{1}$, then we estimate $\left\|x_{n_{0}+1}-\tilde{x}\right\|$. From Lemma 2.7 and (3.1), we have

$$
\begin{aligned}
& \left\|x_{n_{0}+1}-\tilde{x}\right\|^{2} \\
& =\left\|\alpha_{n_{0}} \gamma \phi\left(x_{n_{0}}\right)+\gamma_{n_{0}} x_{n_{0}}+\left[\left(1-\gamma_{n_{0}}\right) I-\alpha_{n_{0}} F\right] \gamma_{n_{0}}-\tilde{x}\right\|^{2} \\
& =\left\|\left[\left(1-\gamma_{n_{0}}\right) I-\alpha_{n_{0}} F\right] \gamma_{n_{0}}-\left[\left(1-\gamma_{n_{0}}\right) I-\alpha_{n_{0}} F\right] \tilde{x}+\alpha_{n_{0}}\left[\gamma \phi\left(x_{n_{0}}\right)-F \tilde{x}\right]+\gamma_{n_{0}}\left(x_{n_{0}}-\tilde{x}\right)\right\|^{2} \\
& =\left\langle\left[\left(1-\gamma_{n_{0}}\right) I-\alpha_{n_{0}} F\right] \gamma_{n_{0}}-\left[\left(1-\gamma_{n_{0}}\right) I-\alpha_{n_{0}} F\right] \tilde{x}+\alpha_{n_{0}}\left[\gamma \phi\left(x_{n_{0}}\right)-F \tilde{x}\right]+\gamma_{n_{0}}\left(x_{n_{0}}-\tilde{x}\right), x_{n_{0}+1}-\tilde{x}\right\rangle \\
& =\left\langle\left[\left(1-\gamma_{n_{0}}\right) I-\alpha_{n_{0}} F\right] \gamma_{n_{0}}-\left[\left(1-\gamma_{n_{0}}\right) I-\alpha_{n_{0}} F\right] \tilde{x}, x_{n_{0}+1}-\tilde{x}\right\rangle+\left\langle\alpha_{n_{0}}\left[\gamma \phi\left(x_{n_{0}}\right)-F \tilde{x}\right], x_{n_{0}+1}-\tilde{x}\right\rangle \\
& +\left\langle\gamma_{n_{0}}\left(x_{n_{0}}-\tilde{x}\right), x_{n_{0}+1}-\tilde{x}\right\rangle \\
& =\left\langle\left[\left(1-\gamma_{n_{0}}\right) I-\alpha_{n_{0}} F\right] \gamma_{n_{0}}-\left[\left(1-\gamma_{n_{0}}\right) I-\alpha_{n_{0}} F\right] \tilde{x}, x_{n_{0}+1}-\tilde{x}\right\rangle+\alpha_{n_{0}}\left\langle\gamma \phi\left(x_{n_{0}}\right)-\gamma \phi(\tilde{x}), x_{n_{0}+1}-\tilde{x}\right\rangle \\
& +\alpha_{n_{0}}\left\langle\gamma \phi(\tilde{x})-F \tilde{x}, x_{n_{0}+1}-\tilde{x}\right\rangle+\gamma_{n_{0}}\left\langle x_{n_{0}}-\tilde{x}, x_{n_{0}+1}-\tilde{x}\right\rangle \\
& \leq\left\|\left[\left(1-\gamma_{n_{0}}\right) I-\alpha_{n_{0}} F\right] \gamma_{n_{0}}-\left[\left(1-\gamma_{n_{0}}\right) I-\alpha_{n_{0}} F\right] \tilde{x}\right\|\left\|x_{n_{0}+1}-\tilde{x}\right\|+\alpha_{n_{0}} \gamma\left\|x_{n_{0}}-\tilde{x}\right\|\left\|x_{n_{0}+1}-\tilde{x}\right\| \\
& +\alpha_{n_{0}}\left\langle\gamma \phi(\tilde{x})-F \tilde{x}, x_{n_{0}+1}-\tilde{x}\right\rangle+\gamma_{n_{0}}\left\|x_{n_{0}}-\tilde{x}\right\|\left\|x_{n_{0}+1}-\tilde{x}\right\| \\
& \leq\left[1-\gamma_{n_{0}}-\alpha_{n_{0}}\left(\eta-\frac{\alpha_{n_{0}} L^{2}}{2\left(1-\gamma_{n_{0}}\right)}\right)\right]\left\|x_{n_{0}}-\tilde{x}\right\|\left\|x_{n_{0}+1}-\tilde{x}\right\|+\alpha_{n_{0}} \gamma\left\|x_{n_{0}}-\tilde{x}\right\|\left\|x_{n_{0}+1}-\tilde{x}\right\| \\
& +\alpha_{n_{0}}\left\langle\gamma \phi(\tilde{x})-F \tilde{x}, x_{n_{0}+1}-\tilde{x}\right\rangle+\gamma_{n_{0}}\left\|x_{n_{0}}-\tilde{x}\right\|\left\|x_{n_{0}+1}-\tilde{x}\right\| \\
& =\left[1-\alpha_{n_{0}}\left(\eta-\frac{\alpha_{n_{0}} L^{2}}{2\left(1-\gamma_{n_{0}}\right)}-\gamma\right)\right]\left\|x_{n_{0}}-\tilde{x}\right\|\left\|x_{n_{0}+1}-\tilde{x}\right\|+\alpha_{n_{0}}\left\langle\gamma \phi(\tilde{x})-F \tilde{x}, x_{n_{0}+1}-\tilde{x}\right\rangle \\
& \leq \frac{1}{2}\left[1-\alpha_{n_{0}}\left(\eta-\frac{\alpha_{n_{0}} L^{2}}{2\left(1-\gamma_{n}\right)}-\gamma\right)\right]\left\|x_{n}-\tilde{x}\right\|^{2}+\frac{1}{2}\left\|x_{n_{0}+1}-\tilde{x}\right\|^{2}+\alpha_{n_{0}}\left\langle\gamma \phi(\tilde{x})-F \tilde{x}, x_{n_{0}+1}-\tilde{x}\right\rangle \\
& \leq \frac{1}{2}\left[1-\alpha_{n_{0}}\left(\eta-\frac{\alpha_{n_{0}} L^{2}}{2\left(1-\gamma_{n}\right)}-\gamma\right)\right]\left(\varepsilon_{0}-\varepsilon\right)^{2}+\frac{1}{2}\left\|x_{n_{0}+1}-\tilde{x}\right\|^{2}+\alpha_{n_{0}}\left\langle\gamma \phi(\tilde{x})-F \tilde{x}, x_{n_{0}+1}-\tilde{x}\right\rangle
\end{aligned}
$$


which implies that

$$
\begin{aligned}
& \left\|x_{n_{0}+1}-\tilde{x}\right\|^{2} \\
< & {\left[1-\alpha_{n_{0}}\left(\eta-\frac{\alpha_{n_{0}} L^{2}}{2\left(1-\gamma_{n_{0}}\right)}-\gamma\right)\right]\left(\varepsilon_{0}-\varepsilon\right)^{2}+2 \alpha_{n_{0}}\left\langle\gamma \phi(\tilde{x})-F \tilde{x}, x_{n_{0}+1}-\tilde{x}\right\rangle } \\
= & {\left[1-\alpha_{n}\left(\eta-\frac{\alpha_{n_{0}} L^{2}}{2\left(1-\gamma_{n_{0}}\right)}-\gamma-M_{n_{0}}\right)\right]\left(\varepsilon_{0}-\varepsilon_{1}\right)^{2} } \\
\leq & \left(\varepsilon_{0}-\varepsilon_{1}\right)^{2}
\end{aligned}
$$

Hence, we have

$$
\left\|x_{n_{0}+1}-\tilde{x}\right\|<\varepsilon_{0}-\varepsilon_{1} .
$$

In the same way, we can get

$$
\left\|x_{n}-\tilde{x}\right\|<\varepsilon_{0}-\varepsilon_{1}, \quad \forall n \geq n_{0} .
$$

It contradict the $\lim \sup _{n \rightarrow \infty}\left\|x_{n}-\tilde{x}\right\| \geq \varepsilon_{0}$.

Case 2. Fixed $\varepsilon_{1}\left(\varepsilon_{1}<\varepsilon_{0}\right)$, if $\left\|x_{n}-\tilde{x}\right\| \geq \varepsilon_{0}-\varepsilon_{1}$ for all $n \geq N \in \mathbb{N}$. In this case from Lemma 2.8, there is a number $r \in(0,1)$, such that

$$
\left\|\phi\left(x_{n}\right)-\phi(\tilde{x})\right\| \leq r\left\|x_{n}-\tilde{x}\right\|, \quad n \geq N .
$$

It follow 3.1 that

$$
\begin{aligned}
& \left\|x_{n+1}-\tilde{x}\right\|^{2} \\
= & \left\langle\left[\left(1-\gamma_{n}\right) I-\alpha_{n} F\right] \gamma_{n}-\left[\left(1-\gamma_{n}\right) I-\alpha_{n} F\right] \tilde{x}, x_{n+1}-\tilde{x}\right\rangle+\alpha_{n}\left\langle\gamma \phi\left(x_{n}\right)-\gamma \phi(\tilde{x}), x_{n+1}-\tilde{x}\right\rangle \\
& +\alpha_{n}\left\langle\gamma \phi(\tilde{x})-F \tilde{x}, x_{n+1}-\tilde{x}\right\rangle+\gamma_{n}\left|x_{n}-\tilde{x}, x_{n+1}-\tilde{x}\right\rangle \\
\leq & {\left[1-\gamma_{n}-\alpha_{n}\left(\eta-\frac{\alpha_{n} L^{2}}{2\left(1-\gamma_{n}\right)}\right)\right]\left\|x_{n}-\tilde{x}\right\|\left\|x_{n+1}-\tilde{x}\right\|+\alpha_{n} \gamma r\left\|x_{n}-\tilde{x}\right\|\left\|x_{n+1}-\tilde{x}\right\| } \\
& +\alpha_{n}\left(\gamma \phi(\tilde{x})-F \tilde{x}, x_{n+1}-\tilde{x}\right\rangle+\gamma_{n}\left\|x_{n}-\tilde{x}\right\|\left\|x_{n+1}-\tilde{x}\right\| \\
\leq & \frac{1}{2}\left[1-\alpha_{n}\left(\eta-\frac{\alpha_{n} L^{2}}{2\left(1-\gamma_{n}\right)}-\gamma r\right)\right]\left\|x_{n}-\tilde{x}\right\|^{2}+\frac{1}{2}\left\|x_{n+1}-\tilde{x}\right\|^{2}+\frac{1}{2}\left\|x_{n+1}-\tilde{x}\right\|^{2}+\alpha_{n}\left\langle\gamma \phi(\tilde{x})-F \tilde{x}, x_{n+1}-\tilde{x}\right\rangle
\end{aligned}
$$

which implies that

$$
\begin{aligned}
& \left\|x_{n+1}-\tilde{x}\right\|^{2} \\
\leq & {\left[1-\alpha_{n}\left(\eta-\frac{\alpha_{n} L^{2}}{2\left(1-\gamma_{n}\right)}-\gamma r\right)\right]\left\|x_{n}-\tilde{x}\right\|^{2}+\alpha_{n}\left[\eta-\frac{\alpha_{n} L^{2}}{2\left(1-\gamma_{n}\right)}-\gamma r\right] \frac{2\left\langle\gamma \phi(\tilde{x})-F \tilde{x}, x_{n+1}-\tilde{x}\right\rangle}{\eta-\frac{\alpha_{n} L^{2}}{2\left(1-\gamma_{n}\right)}-\gamma r} . }
\end{aligned}
$$

Apply Lemma 2.5 to (3.10) to conclude $x_{n} \rightarrow \tilde{x}$ as $n \rightarrow \infty$. It contradict the $\left\|x_{n}-\tilde{x}\right\| \geq \varepsilon_{0}-\varepsilon_{1}$. This completes the proof.

Remark 3. We conclude the article with the following observations.

(i) Theorem 3.2 improve and extend Theorem 3.4 of Marino and Xu [24], Theorem 3.2 of Zhou [8], Theorem 2.1 of Qin [9] and includes those results as special cases. Especially, our results extends above results form contractions to more general MKC. Our iterative scheme studied in this article can be viewed as a refinement and modification of the iterative methods in $[8,9,24]$. On the other hand, our iterative schemes concern an infinite countable family of $k_{i}$-strict pseudo-contractions mappings, in this respect, they can be viewed as an another improvement.

(ii) Our results extend above results form strong positive linear bounded operator to $\eta$-strongly monotone and $L$-Lipschitzian operator. 
(iii) The advantage of the results in this article is that less restrictions on the parameters $\left\{\alpha_{n}\right\},\left\{\beta_{n}\right\},\left\{\gamma_{n}\right\}$ and $\left\{\eta_{i}^{n}\right\}$ are imposed. Our results unify many recent results including the results in $[8,9,24]$.

(iv) It is worth noting that we obtained two strong convergence results concerning an infinite countable family of $\lambda_{i}$-strict pseudo-contractions mappings. Our result is new and the proofs are simple and different from those in $[6,8,9,24,25]$.

\section{Acknowledgements}

The authors are extremely grateful to the referee and the editor for their useful comments and suggestions which helped to improve this article. This research was supported by the National Science Foundation of China (11071169) and the Innovation Program of Shanghai Municipal Education Commission (09ZZ133).

\section{Author details}

${ }^{1}$ Department of Mathematics, Shanghai Normal University, Shanghai 200234,China 2Department of Mathematics, Hubei Normal University, Hubei 435002, China ${ }^{3}$ Department of Mathematics, Shaoxing University, Shaoxing 312000, China ${ }^{4}$ Scientific Computing Key Laboratory of Shanghai University, Shanghai 200234, China

\section{Authors' contributions}

All authors contributed equally and significantly in writing this paper. All authors read and approved the final manuscript.

\section{Competing interests}

The authors declare that they have no competing interests.

Received: 29 October 2011 Accepted: 23 March 2012 Published: 23 March 2012

\section{References}

1. Stampacchia, G: Formes bilineaires coercitives surles ensembles convexes. C R Acad Sci de Paris. 258, 4413-4416 (1964)

2. Yamada, I: The hybrid steepest-descent method for variational inequality problems over the intersection of the fixed point sets of nonexpansive mappings. Stud Comput Math. 8, 473-504 (2001)

3. Noor, MA: Some developments in general variational inequalities. Appl Math Comput. 152, 199-277 (2004). doi:10.1016/ S0096-3003(03)00558-7

4. $\mathrm{Xu}, \mathrm{HK}, \mathrm{Kim}, \mathrm{TH}$ : Convergence of hybrid steepest-descent methods for variational inequalities. J Optim Theory Appl. 119, 185-201 (2003)

5. Glowinski, R: Numerical Methods for Nonlinear Variational Problems. Springer, New York (1984)

6. Yao, Y, Chen, RD, Yao, JC: Strong convergence and certain control conditions for modified Mann iteration. Nonlinear Anal. 68, 1687-1693 (2008). doi:10.1016/j.na.2007.01.009

7. Moudafi, A: Viscosity approxiamtion methods for fixed points problems. J Math Anal Appl. 241, 46-55 (2000). doi:10.1006/jmaa.1999.6615

8. Zhou, HY: Convergence theorems of fixed points for k-strict pseudo-contractions in Hilbert space. No-linear Anal. 69, 456-462 (2008)

9. Qin, $X L$, Shang, M, Kang, SM: Strong convergence theorems of modified Mann iterative process for strict pseudocontractions in Hilbert spaces. Nonlinear Anal. 70(3):1257-1264 (2009). doi:10.1016/j.na.2008.02.009

10. Banach, S: Sur les opérations dans les ensembles abstraits et leur application aux équations intégrales. Fund Math. 3, 133-181 (1922)

11. Meir, A, Keeler, E: A theorem on contraction mappings. J Math Anal Appl. 28, 326-329 (1969). doi:10.1016/0022-247X (69) $90031-6$

12. Browder, FE, Petryshyn, WV: Construction of fixed points of nonlinear mappings in Hilbert space. J Math Anal Appl. 20, 197-228 (1967). doi:10.1016/0022-247X(67)90085-6

13. Suzuki, T: Strong convergence of Krasnoselskii and Manns type sequences for one-parameter nonexpansive semigroups without Bochner integrals. J Math Anal Appl. 305(1):227-239 (2005). doi:10.1016/j.jmaa.2004.11.017

14. Xu, HK: An iterative approach to quadratic optimization. J Optim Theory Appl. 116, 659-678 (2003). doi:10.1023/ A:1023073621589

15. Yao, YH, Liou, YC, Kang, SM: Two-step projection methods for a system of variational inequality problems in Banach spaces. J Global Optim (2011). doi:10.1007/s10898-011-9804-0

16. Yao, YH, Liou, YC, Kang, SM: Iterative methods for $k$-strict pseudo-contractive mappings in Hilbert spaces. An St Univ Ovidius Constanta. 19, 313-330 (2011)

17. Yao, YH, Noor, MA, Liou, YC: Strong convergence of a modified extra-gradient method to the minimum-norm solution of variational inequalities. Abstr Appl Anal 2012, 9 (2012). Article ID 817436

18. Yao, YH, Chen, RD, Liou, YC: A unified implicit algorithm for solving the triple-hierarchical constrained optimization problem. Math Comput Model. 55, 1506-1515 (2012). doi:10.1016/j.mcm.2011.10.041

19. Yao, YH, Cho, YJ, Liou, YC: Hierarchical convergence of an implicit double-net algorithm for non-expansive semigroups and variational inequalities. Fixed Point Theory Appl (2011). doi:10.1186/1687-1812-2011-101

20. Yao, YH, Noor, MA, Liou, YC, Kang, SM: Iterative algorithms for general multi-valued variational inequalities. Abstr Appl Anal 2012, 10 (2012). Article ID 768272, doi:10.1155/2012/768272

21. Yao, YH, Shahzad, N: Strong convergence of a proximal point algorithm with general errors. Optim Lett (2011). doi:10.1007/s11590-011-0286-2 
22. Yao, YH, Wu, JG, Liou, YC: Regularized methods for the split feasibility problem. Abstr Appl Anal 2012, 13 (2012). Article ID 140679, doi:10.1155/2012/140679

23. Suzuki, T: Moudafi's viscosity approximations with Meir-Keeler contractions. J Math Anal Appl. 325, 342-352 (2007). doi:10.1016/j.jmaa.2006.01.080

24. Marino, G, Xu, HK: A general iterative method for nonexpansive mappings in Hilbert spaces. J Math Anal Appl. 318, 43-52 (2006). doi:10.1016/j.jmaa.2005.05.028

25. Cai, G, Hu, CS: Strong convergence theorems of a general iterative process for a finite family of $\lambda_{i}$-strict pseudocontractions in q-uniformly smooth Banach spaces. Comput Math Appl. 59, 149-160 (2010). doi:10.1016/j. camwa.2009.07.068

doi:10.1186/1687-1812-2012-46

Cite this article as: Song et al: Strong convergence of a new general iterative method for variational inequality problems in Hilbert spaces. Fixed Point Theory and Applications 2012 2012:46.

\section{Submit your manuscript to a SpringerOpen ${ }^{\odot}$} journal and benefit from:

- Convenient online submission

- Rigorous peer review

- Immediate publication on acceptance

- Open access: articles freely available online

- High visibility within the field

- Retaining the copyright to your article

Submit your next manuscript at $\boldsymbol{s p r i n g e r o p e n . c o m ~}$ 